\title{
Schweder, Marcel
}

\section{Lehrerarbeit im Strafvollzug - Ein Diskurs aus historischer Sicht}

Seifried, Jürgen [Hrsg.]; Faßhauer, Uwe [Hrsg.]; Seeber, Susan [Hrsg.]: Jahrbuch der berufs- und wirtschaftspädagogischen Forschung 2014. Opladen [u.a.] : Verlag Barbara Budrich 2014, S. 39-52. (Schriftenreihe der Sektion Berufs- und Wirtschaftspädagogik der Deutschen Gesellschaft für Erziehungswissenschaft (DGfE); 217)

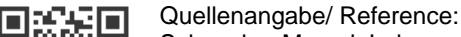

Schweder, Marcel: Lehrerarbeit im Strafvollzug - Ein Diskurs aus historischer Sicht - In: Seifried, Jürgen [Hrsg.]; Faßhauer, Uwe [Hrsg.]; Seeber, Susan [Hrsg.]: Jahrbuch der berufs- und wirtschaftspädagogischen Forschung 2014. Opladen [u.a.] : Verlag Barbara Budrich 2014, S. 39-52 URN: urn:nbn:de:0111-pedocs-97249 - DOI: 10.25656/01:9724

https://nbn-resolving.org/urn:nbn:de:0111-pedocs-97249

https://doi.org/10.25656/01:9724

in Kooperation mit / in cooperation with:

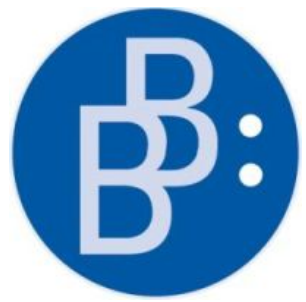

https://www.budrich.de

\section{Nutzungsbedingungen}

Dieses Dokument steht unter folgender Creative Commons-Lizenz:

http://creativecommons.org/licenses/by-nc-nd/3.0/de/deed - Sie dürfen das Werk bzw. den Inhalt unter folgenden Bedingungen vervielfältigen, verbreiten und öffentlich zugänglich machen: Sie müssen den Namen des Autors/Rechteinhabers in der von ihm festgelegten Weise nennen. Dieses Werk bzw. dieser Inhalt darf nicht für kommerzielle Zwecke verwendet werden und es darf nicht bearbeitet, abgewandelt oder in anderer Weise verändert werden.

Mit der Verwendung dieses Dokuments erkennen Sie die Nutzungsbedingungen an.

\section{Terms of use}

This document is published under following Creative Commons-License: http://creativecommons.org/licenses/by-nc-nd/3.0/de/deed.en - You may copy, distribute and transmit, adapt or exhibit the work in the public as long as you attribute the work in the manner specified by the author or licensor. You are not allowed to make commercial use of the work or its contents. You are not allowed to alter, transform, or change this work in any other way.

By using this particular document, you accept the above-stated conditions of use.

\section{Kontakt / Contact:}

\section{peDOCS}

DIPF | Leibniz-Institut für Bildungsforschung und Bildungsinformation Informationszentrum (IZ) Bildung

E-Mail: pedocs@dipf.de

Internet: www.pedocs.de

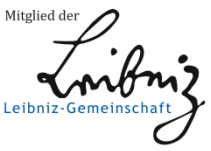




\section{Jahrbuch der berufs- und wirtschaftspädagogischen Forschung 2014}

Jürgen Seifried, Uwe Faßhauer Susan Seeber (Hrsg.)

DGE Deutsche Gesellschaft 
Schriftenreihe der Sektion

Berufs- und Wirtschaftspädagogik der Deutschen Gesellschaft für Erziehungswissenschaft (DGfE) 
Jürgen Seifried

Uwe Faßhauer

Susan Seeber (Hrsg.)

\section{Jahrbuch der berufs- und wirtschaftspädagogischen Forschung 2014}

Verlag Barbara Budrich

Opladen • Berlin • Toronto 2014 
Bibliografische Information der Deutschen Nationalbibliothek

Die Deutsche Nationalbibliothek verzeichnet diese Publikation in der Deutschen Nationalbibliografie; detaillierte bibliografische Daten sind im Internet über http://dnb.d-nb.de abrufbar.

(C) Dieses Werk ist im Verlag Barbara Budrich erschienen und steht unter folgender Creative Commons Lizenz: http://creativecommons.org/licenses/by-nc-nd/3.0/de/ Verbreitung, Speicherung und Vervielfältigung erlaubt, kommerzielle Nutzung und Veränderung nur mit Genehmigung des Verlags Barbara Budrich.

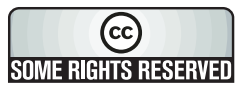

Dieses Buch steht im OpenAccess Bereich der Verlagsseite zum kostenlosen Download bereit (http://dx.doi.org/10.3224/84740164)

Eine kostenpflichtige Druckversion (Printing on Demand) kann über den Verlag bezogen werden. Die Seitenzahlen in der Druck- und Onlineversion sind identisch.

$$
\begin{array}{ll}
\text { ISBN } & 978-3-8474-0164-3 \text { (Paperback) } \\
\text { eISBN } & \mathbf{9 7 8 - 3 - 8 4 7 4 - 0 4 4 1 - 5 ~ ( e B o o k ) ~} \\
\text { DOI } & 10.3224 / 84740164
\end{array}
$$

Umschlaggestaltung: bettina lehfeldt graphic design, Kleinmachnow

Typografisches Lektorat: Judith Henning, Hamburg

Verlag Barbara Budrich, http://www.budrich-verlag.de 


\section{Inhaltsverzeichnis}

Vorwort 9

\section{Teil I: Perspektiven der historischen Berufsbildungsforschung}

Frank-Lothar Kroll

Möglichkeiten und Notwendigkeiten historiographischen Arbeitens in der Berufs- und Wirtschaftspädagogik........................................................ 1

Volker Bank, Annekathrin Lehmann

Theodor Franke. Sächsischer Pionier wirtschaftspädagogischen

Denkens in Deutschland

Marcel Schweder

Lehrerarbeit im Strafvollzug - Ein Diskurs aus historischer Sicht

\section{Teil II: Kompetenzmodellierung, -messung und -förderung}

Eveline Wittmann, Ulrike Weyland, Annette Nauerth, Ottmar Döring, Simone Rechenbach, Julia Simon, Iberé Worofka Kompetenzerfassung in der Pflege älterer Menschen - Theoretische und domänenspezifische Anforderungen der Aufgabenmodellierung 53

Simon Heinen, Martin Frenz, Christopher M. Schlick

Bildung für nachhaltige Entwicklung in der Gebäudeenergieberatung - Entwicklung eines Kompetenzmodells für die Förderung reflexiver Handlungsfähigkeit

Diana Stuckatz, Cornelia Wagner

Qualifizierungsangebote in der Pflegehilfe für Personen mit geringen Grundbildungskenntnissen - Empirische Studien zur Entwicklung von Lehr-Lern-Umgebungen und Arbeitsmaterialien. 81 


\section{Teil III: Gestaltung und Analyse von Lehr-Lern-Prozessen}

Eva Höpfer, Andrea Reichmuth, Doreen Holtsch, Franz Eberle

Wer sieht was? - Zum Umgang mit unterschiedlichen Sichtweisen auf

Unterricht am Beispiel des kaufmännischen Berufsschulunterrichts 95

Mandy Hommel

Sozial geteilte Reflexion - eine explorative Studie im

Mathematikunterricht. 109

Gerhard Minnameier, Rico Hermkes

„Kognitive Aktivierung“ und „konstruktive Unterstützung“ als Lehr-

Lern-Prozess-Größen - Eine Konzeption im rechnungswesen-

didaktischen Kontext

\section{Teil IV: Lehrerbildung und pädagogische Professionalität}

Nicole Kimmelmann, Johannes Lang

Lehramtsstudierende mit Migrationshintergrund und ihre

Schwierigkeiten an der Universität

Robert W. Jahn

Stützlehrer als neuer pädagogischer Profi in der Beruflichen Bildung?! .... 147

Sabrina Berg

Pädagogische Praxis und Reproduktion sozialer Ungleichheit - zur

Berücksichtigung sozialer Herkunft im Wirtschaftsunterricht..... 161

\section{Teil V: Perspektiven der Berufsbildungsforschung}

\section{Miriam Voigt}

Neo-institutionalistische und mikropolitische Prozesse in

Schulentwicklungsprojekten

Lara Forsblom, Lucio Negrini, Jean-Luc Gurtner \& Stephan Schumann

Lehrvertragsauflösungen und die Rolle der betrieblichen Auswahl von

Auszubildenden 
Marius R. Busemeyer

Organisierte Interessen, Parteipolitik und institutioneller Wandel im deutschen Berufsbildungssystem

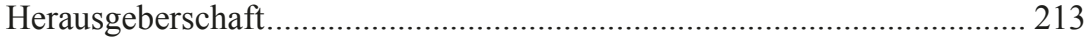

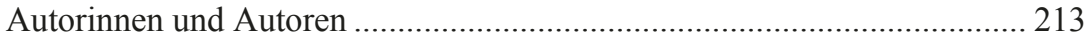




\title{
Lehrerarbeit im Strafvollzug - Ein Diskurs aus historischer Sicht
}

\author{
Marcel Schweder
}

\section{Verschlossenes erschließen - Abgrenzung und Zielstellung}

„Er hatte diese Freude so wenig, und es that ihm so weh, wenn er nach aller Arbeit nichts ausrichtete, und nach langen vergebenen Hoffnungen sehen mußte, daß mit einem Menschen gar nichts zu machen war " (Pestalozzi).

Um etwaigen Missverständnissen vorzubeugen: die nachfolgenden Ausführungen können und sollen nicht dazu dienen, die Lehrerarbeit im Strafvollzug umfassend zu beschreiben. Dies ist, wie sich hoffentlich zeigen wird, auch nicht ohne weiteres möglich. Ziel ist vielmehr, die Entstehung der Gefängnisschule sowie die unweigerlich daran gebundene Etablierung der Lehrerarbeit vor dem Hintergrund der institutionalisierten Idee, Taten durch den Entzug der Freiheit zu ahnden, zu beschreiben. Die Historie als Hintergrundfolie erscheint diesbezüglich insofern zielführend, als dadurch die Bedeutung und die Bedingungen der Lehrerarbeit hinter Gittern, insbesondere das tradierte professionelle Selbstverständnis, besonders deutlich werden.

Ausgehend vom Freiheitsentzug als ,zeitgemäße“ Form von Strafe wird der Gefangenenunterricht als Mittel der Besserung in seinem historischen Entstehungsprozess und der damit verbundenen Intention beschrieben. Beide Ausführungen verfolgen das Ziel, den Zugang zu einer Arbeitswelt zu ermöglichen, welche für Außenstehende sonst - im Sinne des Wortes - verschlossen bleibt. In der Folge kann eine klare Vorstellung darüber entwickelt werden, wodurch sich die Professionalität der Lehrenden konstituiert bzw. welche spezifischen Anforderungen für die Lehrenden aus der Arbeitsstätte selbst, sprich der „totalen Institution“ Gefängnis, den Mechanismen und der Logik eines institutionalisierten Entzugs der Freiheit sowie aus den individuellen Dispositionen der Gefangenen ${ }^{1}$ resultieren.

Anzumerken bleibt, dass mit dem vorliegenden Beitrag vorrangig intendiert wird, das Wesen der Lehrerarbeit hinter Gittern mit Hilfe von eher explorativ erworbenen - so war der Verfasser selbst im Jugendstrafvollzug als

1 Wenngleich die Ausführungen auf Quellen basieren, welche den männlichen Gefangenen im Blick hatten, so unterliegen die Schlussfolgerungen dieser Einschränkung nicht. Insbesondere die Konzeption von Bildungsmaßnahmen kann/sollte m. E. nicht aus einer geschlechtsspezifisch geprägten Sichtweise heraus gedacht werden, wenngleich gewisse Konstellationen (Schwangerschaft) die Umsetzung dieses Grundsatzes verhindern können. 
Lehrer tätig - und weniger empirisch begründeten Erkenntnissen zu beschreiben und damit prinzipiell die Frage aufzuwerfen, ob die Lehrerarbeit im Strafvollzug nicht stärker zum (Forschungs-)Gegenstand der Lehrerbildung werden müsste.

Insofern wäre viel getan, wenn die Ausführungen einen Beitrag dazu leisten, ein realistisches und vorurteilsfreies Bild über die Lehrerarbeit hinter Gittern zu zeichnen sowie, nicht zuletzt, einen Diskurs zur Notwendigkeit der Professionalisierung der Lehrenden im Strafvollzug anzuregen.

\section{Der Freiheitsentzug als ultima ratio}

Die Verdrängung der Todesstrafe und damit die Favorisierung des Freiheitsentzugs, im Übergang vom 16. zum 17. Jahrhundert, gehen einher mit dem sich ausweitenden Kapitalismus. Unter den neuen Vorzeichen, insbesondere unter dem Aspekt des Arbeitskräftemangels, änderten sich die Einstellungen bezüglich der zielführendsten Form von Strafe. Im Gefangenen wurde nunmehr nicht mehr nur der Abtrünnige, sondern auch (s)ein ökonomisches Kapital gesehen. Diesem Verständnis folgend, wurde die Todesstrafe und damit der Verlust einer Arbeitskraft dem erwirtschaftbaren Gewinn mit selbiger im Zeitraum der Freiheitsstrafe gegengerechnet: ,...was nutzet ein Dieb, der um funfftzig Gulden ist gehenckt worden, sich oder diesem, dem er gestohlen? da er doch im Werckhauß in einem Jahr wol viermal so viel wieder verdienen kan...?“ (Becher zit. n. Cornel 1984, S. 22). Insofern war der Freiheitsentzug als Alternative zur Leibes- und Todesstrafe im doppelten Sinne zielführender. Einerseits konnten die Seelen der Verurteilten durch die Arbeit "gerettet" (Deimling 1969, S. 7) werden, andererseits stellte der Strafvollzug kostengünstige Arbeitskräfte zur Verfügung.

Mit der beginnenden Industrialisierung und der damit verbundenen, notwendigen Technisierung waren die Arbeitshäuser jedoch nicht mehr konkurrenzfähig (vgl. Eberle 1980, S. 35). Zudem veränderte sich die Auffassung, ausschließlich über die Arbeit zu erziehen. Im Kontext der ökonomischen und politischen Wirren des ausgehenden 18. sowie des beginnenden 19. Jahrhunderts erlebte die Leibesstrafe zwar eine Art Renaissance, allerdings und bezeichnender Weise als Funktion der Freiheitsstrafe. „Die Gesundheit der Bestraften wurde durch die in diesen Gefängnissen vollzogene Freiheitsstrafe mehr zu Grunde gerichtet als durch die Leibesstrafen des alten Rechts, und an der Gefängniskrankheit starben mehr Gefangene als unter dem früheren Recht am Galgen oder auf dem Schafott" (Krohne 1901 zit. n. Cornel 1984, S. 24). Dieser Rückfall sollte die Etablierung des Freiheitsentzugs als generelle Strafmaßnahme jedoch nicht verhindern. Denn gerade für eine Gesellschaft, welche auf der individuellen Freiheit die eigene Existenz begründet, 
erscheint die Einfachheit des Gefängnisses eben darin zu liegen, die Gefangenen dieses Gutes zu berauben (vgl. Foucault 1994, S. 296). Legitimiert wurde die Freiheitsstrafe nunmehr durch den Gedanken der Besserung, zunehmend jedoch unter neuem Vorzeichen. So war die Gefängnisstrafe ,immer schon eine ,legale Haft' mit dem Zweck der Besserung bzw. ein Unternehmen zur Veränderung von Individuen“ (Foucault 1994, S. 297f.). Seit dem Beginn des 20. Jahrhunderts gewinnt allerdings eher die „Behandlungsfunktion“ (Bildungsmaßnahmen, Therapien u. Ä.) gegenüber der „Besserungsfunktion“ an Bedeutung. ${ }^{2}$

Die Einführung der Freiheitsstrafe als neue ultima ratio hat somit nicht nur den Weg für eine Neuinterpretation der notwendigen Behandlung und Bestrafung von Verbrechern geebnet, sie ist zudem Ursprung für Bildungsmaßnahmen und damit für den Unterricht im Gefängnis.

\section{Gefangenenunterricht - Mittel zu welchem Zweck?}

Der Ansatz, dass Gefängnisunterricht einen positiven Einfluss auf die Entwicklung und Sozialisation von Inhaftierten hat, ist nicht neu. Seinen Ursprung hat dieser Gedanke im 16. Jahrhundert, im Zusammenhang mit der Entstehung der ersten Zucht- und Arbeitshäuser (Eberle 1983, Wittig 1939). Im Zuge der sich verändernden wirtschaftlichen und sozialen Entwicklungen, den damit einhergehenden sozialen Problemen sowie dem neuen Verständnis über die Lebensaufgabe bedurfte es neuer disziplinierender Institutionen (vgl. Marzahn/Ritz 1984, S. 15ff.). Ausgehend vom religiösen Verständnis des Calvinismus, in welchem Beruf und Arbeit von zentraler Bedeutung für eine religionskonforme Lebensbewältigung waren, lag die Aufgabe der Zuchtund Arbeitshäuser in der Erziehung zur Arbeit (vgl. Eberle 1983, S. 440, Deimling 1969, S. 6ff.). Der bevorzugte Unterricht war damit konsequenterweise Religionsunterricht, ${ }^{3}$ da nur über diesen die Logik der Buße und damit der „Rettung“ (Deimling 1969, S. 7) verwirklicht werden konnten. „Dem Elementarunterricht, wenn er überhaupt erteilt wurde, kam eine bloße instrumentelle Funktion zu; er war lediglich Mittel zum eigentlichen Zweck: Damit

2 An dieser Stelle sei darauf hingewiesen, dass die positive Besetzung des Begriffs der Behandlung nicht für die Zeit des Nationalsozialismus gelten kann. Dem Grundsatz der Auslese folgend, sollten derartige Maßnahmen nur dort Einsatz finden, ,wo Einsicht und Hoffnung besteht, daß der Gefangene wieder in die Volksgemeinschaft eingegliedert werden kann“ (Gürtner 1937, zit. n. Eberle 1980, S. 61). Die politisch-ideologische Basis für den Strafvollzug war damit das Rasse- und Ausleseprinzip, was letztlich ebenso an der Ausgestaltung der Maßnahmen, wie dem Gefängnisunterricht (vgl. Kap. 3), abzulesen ist.

3 Dieser Auffassung folgend war auch eine Separierung der Gefangenen nicht nötig, da alle Problemgruppen das gleiche Merkmal, nämlich die „Unfähigkeit zur eigenständigen Reproduktion“"(Marzahn/Ritz 1984, S. 30), verband. 
die Gefangenen lesen konnten und fähig waren, mit den biblischen Wahrheiten umzugehen und sie in rechter Weise aufzunehmen, mußten sie lesen und schreiben können“" (Eberle 1983, S. 440). In der Manifestierung eben dieser Auffassung sieht Eberle (ebd.) die Ursache für die, bis in Mitte des 20. Jahrhunderts erhaltene, Dominanz der Kirche im Strafvollzug und die damit verbundene Ausrichtung der Bildung.

Die Gefängnisschule wird im 19. Jahrhundert zu einem festen Bestandteil der Strafanstalten (Eberle 1983, Wittig 1939). Im Zuge der sich wandelnden Sichtweise und der Favorisierung des „Besserungsgedankens“ (Wittig 1939, S. 87), demzufolge die Straftäter zu bessern/zu erziehen sind (vgl. auch Krell 1873, Pestalozzi 1936) und nicht allein ihre Tat zu sühnen ist, wurde auch dem Unterricht zunächst eine höhere Bedeutung beigemessen. Dennoch stand man der Etablierung eines Schulbetriebs im öffentlichen Sinne zunehmend konträr gegenüber. Selbst Johann Hinrich Wichern, welcher als einer der wichtigsten Wegbereiter bezüglich der Etablierung des Gefängnisunterrichts gilt (vgl. Reinheckel 2008, S. 205, Myschker/Hoffmann 1984, S. 16), schien es für notwendig zu erachten, im „Erlaß wegen des Schulunterrichts in den Strafanstalten (1860)“ seinen Standpunkt bezüglich der originären Funktion des Strafvollzugs in Abgrenzung zum Gefängnisunterricht deutlich zu machen. „Dadurch wird [...] dem etwaigen Mißverständnis vorgebeugt werden, als ob die Strafanstalten für die Zukunft in Schulanstalten verwandelt werden sollten. Davon kann nicht die Rede sein. Die Strafanstalten müssen vielmehr das, was ihr Name sagt, verbleiben“ (Hervorhebung im Original; Wichern 1860, S. 179). Behringer stützt seine Position auf den Gedanken, „dass es als eine soziale Ungerechtigkeit betrachtet werden muss, dem Gliede der Gesellschaft, welches deren Gesetze mit Füssen getreten hat, die nicht genug zu schätzende Wohlthat einer Weiterbildung in den Schulkenntnissen gleichsam als Belohnung zuzugestehen oder gar aufzunötigen, während der vor dem Gesetze sittlich intakte Mensch Fortbildungsunterricht bezahlen muss, wenn er ihn überhaupt erhalten kann“ (Behringer 1901, S. 107). Beide sehen zwar die Notwendigkeit von Unterricht als gegeben, allerdings eher unter dem Aspekt der sittlichen Hebung und weniger unter dem einer umfassenden Vermittlung von Kenntnissen. Schule im Strafvollzug ist demzufolge „nur berechtigt, wenn die geistige Entwicklung des Schülers noch auf einer so tiefen Stufe steht, dass er am Besserungsunterricht nicht mit Nutzen teilnehmen kann“ (ebd., S. 109). Krell sah insbesondere in der Einrichtung von Gewerbeschulen ,eine Übertreibung, die zugleich ein schreiendes Unrecht gegen den freien ehrlichen Arbeiter involvirt" (1873, S. 81). Lombroso (zit. nach Ellger 1922, S. 91) lehnt den Schulunterricht ganz ab, da „elementare Geistesbildung [...] ein Mittel mehr in der Hand des Verbrechers [ist, M. S.], sich im Verbrechen zu vervollkommnen und zu rezidivieren". In der Folge tritt die Idee einer Gefängnisschule nach dem Vorbild der Volksschule wieder hinter die Arbeit als erzieherisches und ökonomisch wertvolleres Mit- 
tel zurück. Schule und Bildung wird zum freiwilligen Gut, welches in der Freizeit anzueignen ist.

Mit dem beginnenden 20. Jahrhundert kam es zum wiederholten Mal zum Paradigmenwechsel. Aus der reformierten Auffassung von Erziehung heraus bekam der Strafvollzug einen „sozialpädagogischen“ Auftrag. Die demokratische Werteordnung der Republik verlangte nach der Einbeziehung der Inhaftierten, da dies für den Erziehungsprozess und eine gelingende Sozialisation notwendig erschien. Die Notwendigkeit der Bildungsmaßnahmen schien insbesondere unter dem Gesichtspunkt der Fürsorgepflicht unabdingbar zu sein (vgl. Eberle 1983). Zudem beförderte der vermutete Zusammenhang zwischen Bildung und Delinquenz die Reformierung der Schule im Strafvollzug. So bildete der Grundsatz der aktiven Beteiligung der Inhaftierten am Erziehungsprozess für Bondy (1925) und Hermann (1923) den Ausgangspunkt für ihre (Forschungs)Aktivitäten in der Hamburger Jugendstrafanstalt Hahnöfersand.

Im sich anschließenden Nationalsozialismus wurde das neue Volksverständnis (Deutschtum) gleichsam auf den Strafvollzug sowie die Gefängnisschule übertragen. Übergeordnetes Ziel der Gefängnisschule zur Zeit des Nationalsozialismus war demnach der ,,deutsche Mensch“ (Wittig 1939, S. 135). In der Konsequenz bekam der Unterricht abermals eine neue inhaltliche Funktion, er diente nicht mehr vorrangig der Entwicklung einer allgemeinen Grundbildung, sondern der Schulung der neuen Weltanschauung (vgl. Eberle 1983, S. 444). Anzumerken bleibt, dass der Erziehungsgedanke zwar nicht aufgegeben, mit dem Ziel der Gesinnungs- und Charakterbildung sowie dem Blick auf die Volksgemeinschaft allerdings völlig neu besetzt wurde.

In den ersten Jahrzehnten nach 1945 kam es, in Bezug auf die Gesetzgebung (Formulierung), wider Erwarten zu keinem ,radikalen Bruch mit der nationalsozialistischen Vergangenheit" (ebd.) und damit auch zu keiner Reformierung der Bildungsmaßnahmen im Strafvollzug. Mit Blick auf die 60er und 70er Jahre verweist Müllges (1996, S. 278) auf „die desolate Stellung des Unterrichts in JVA, der eher als Beschäftigungstherapie für die letzten freien Stunden dient denn als planmäßige Veranstaltung des Lehrens und Lernens zum Zweck der Resozialisierung“". Rückblickend kommt der nordrhein-westfälische Justizminister 1973 zur gleichen Erkenntnis (zit. nach Eberle 1983, S. 445): „Es fehlte eine Systematik der Angebote an Kursen... [und, M. S.] [...] eine klare Zielvorstellung [bezüglich, M. S.] des Unterrichts im Strafvollzug“. Je nach Strafanstalt variierte der Unterricht zwischen „Aktualitätenunterricht" (Deimling 1969, S. 257) sowie moralischer Belehrungsmaßnahme, wobei die individuellen Problemlagen der Gefangenen unberücksichtigt blieben (vgl. Hoffmann 1989, S. 466). Gegen Ende des 20. Jahrhunderts verfestigte sich auch im Strafvollzug die Auffassung, dass sich die Kategorien Bildung und Erziehung, mit ihren Zielperspektiven Wissen und 
Verhalten, weder getrennt betrachtet noch voneinander losgelöst umgesetzt werden können.

Schlussendlich wurden die Bedeutung und die Notwendigkeit sozialpädagogisch ausgerichteter Bildungsmaßnahmen für das Erreichen des übergeordneten Vollzugsziels (Leben in sozialer Verantwortung und ohne Straftaten) erkannt. In den Blick geriet damit aber (wieder) die Frage nach der Professionalität und der Stellung der Lehrenden im Strafvollzug, auf deren Basis sich ein einheitliches Berufsbild konstituieren ließe. ${ }^{4}$ Die Unbestimmtheit der beruflichen Identität ist gebunden an die teilweise komplementären Vorstellungen zum „Berufs- und Funktionsbild“ (Busch 1991, S. 259) der Lehrer im Freiheitsentzug sowie die Zugehörigkeit zum Disziplinierungsapparat selbst.

\section{Lehrerarbeit hinter Gittern}

\subsection{Die Suche nach einem professionellen Selbstverständnis}

Wenngleich die Lehrenden im Strafvollzug zu den ältesten pädagogischen Berufen (vgl. Deimling 1971, S. 53) zählen, so hat es dieser Berufsstand doch nie geschafft, ein professionelles Selbstverständnis bezüglich seiner Arbeit zu entwickeln. Insbesondere die (scheinbar notwendige) Quantifizierbarkeit des eigenen Erfolgs stellt für die Lehrenden im Strafvollzug immer noch die (einzige) Kernkategorie ihres beruflichen Handelns dar (vgl. Borchert 2007, S. 284ff.). Damit folgen sie der Grundlogik des Freiheitsentzugs und sind mit dieser gewissermaßen ungewollt konform, was durchaus als Indiz für die Verstrickung von Bildungsarbeit und Disziplinierungsapparat (vgl. Eberle 1983, S. 439) gesehen werden kann. Während sich die Einfachheit des Freiheitsentzuges darin begründet, dass sie eine ,exakte Quantifizierung der Strafe nach der Variable Zeit" (Foucault 1994, S. 297) erlaubt, gestattet die Variable (formaler) Abschluss, im Kontext der Bildungsmaßnahmen im Vollzug und aus Sicht der Lehrenden, eine exakte Quantifizierung des Erfolgs. ${ }^{5}$

4 Bis heute ist es bei wenigen Versuchen und Ansätzen geblieben (vgl. Deimling 1971, Busch 1991, Bierschwale 1994). Einzig die Bundesarbeitsgemeinschaft der Lehrerinnen und Lehrer im Justizvollzug (2001, S. 25) hat bislang einen umfassenderen (explorativ) Entwurf zum Berufsbild vorgelegt.

5 Das diese Quantifizierung nur scheinbar eine ,klare Sache“ (Foucault 1994, S. 297) ist, beschreibt und erörtert Wagner (1984) sehr ausführlich in seinem Buch „Das absurde System“. Insofern unterliegt nicht nur Foucault, sondern auch die Lehrenden dem Irrglauben, dass sich mit einer definierten Variable die Zielfunktion des Freiheitsentzugs beziehungsweise die der didaktisch-pädagogischen Handlung beschreiben lässt. 
Das Fehlen einer eigenen klar umrissenen Beruflichkeit und damit eines Berufsbildes lässt sich einerseits auf die historische Genese des Berufsstandes, andererseits auf die Stellung der Lehrenden im Vollzug zurückführen. Im 17. und 18. Jahrhundert entsprach der Lehrer im Strafvollzug dem Anstaltsgeistlichen. „Die starke Verwurzelung der Geistlichen in der Anstaltsschule hat ihre Ursache darin, daß mit dem Aufkommen des generellen Freiheitsentzugs der Religionsunterricht fester Bestandteil des Anstaltslebens war, daß die Lehrer zunächst nur Gehilfen der Geistlichen waren und diese nach der Einführung des profanen Unterrichts die unmittelbare Schulaufsicht über die Lehrer erhielten“" (Eberle 1984, S. 64). Sie hatten somit nicht nur keinen Einfluss auf die Gestaltung des Strafvollzugs und seiner Maßnahmen, sondern waren nicht mehr als „säkularisierte Seelsorger“ (Eberle 1980, S. 156). Wenngleich der Religionsunterrichts und damit die Anstaltsgeistlichen gegen Mitte des 19. Jahrhunderts, infolge der wachsenden Bedeutung des weltlichen "Schulunterrichts bei der Verwirklichung des Besserungsprinzips“ (Eberle 1983, S. 440) in den Hintergrund gerieten, so ist die Kirche als Institution im Strafvollzug erhalten geblieben. Alles in allem führte aber die verlorengegangene dominierende Position der Anstaltsgeistlichen zur Aufwertung und Stärkung der Lehrenden.

Die Hauptaufgabe der Lehrenden im Strafvollzug bestand nunmehr darin, die Gefangenen zu erziehen und ihnen Vorbild zu sein. Dies geschah zunächst noch unter dem Vorzeichen einer Lernschule, das heißt der Vermittlung von Elementarkenntnissen, später, innerhalb der Weimarer Republik, insbesondere aus dem Verständnis einer Erziehungsschule heraus. Konterkariert wurde die Idee der Fürsorge- und Vorbildfunktion vor allem durch den Einsatz von Gefangenen als Lehrende, ,die nach diesem Verständnis wohl kaum die gewünschten vorbildlichen Charaktere abgeben konnten“" (Eberle 1980, S. 157). Dessen ungeachtet wurde die Idee, Gefangene als Lehrkräfte einzusetzen, nie wirklich aufgegeben. Diese Sichtweise begründet sich einerseits darauf, dass der Gefangene die Lebenswelt und die Problemlagen seiner Mithäftlinge kennt und daher aus motivationaler und didaktischer Sicht eher Zugang zu diesen bekommt, andererseits könnte so das „brachliegende Potential“" (Silber 2000, S. 177) der Gefangenen besser genutzt werden. ${ }^{6}$ Die unklare Position sowie das diffuse Aufgabenfeld innerhalb der Institution Gefängnis gab den Lehrenden damit zu keiner Zeit Ansatzpunkte bezüglich der Herausbildung einer eigenen Professionalität. Wenngleich die Dominanz der Kirche und damit die des Anstaltsgeistlichen bereits vor der Weimarer Zeit zurückgedrängt wurde, was die Lehrenden in ihrer Position gestärkt hat, so blieb weiterhin ein Aspekt der Professionalisierung ungelöst: Ihre formale Qualifizierung für den Strafvollzug.

6 Silber geht sogar so weit, dass ehemaligen Insassen nach ihrer Bewährung die Möglichkeit gegeben werden müsste, ,eine pädagogische Ausbildung zu machen um anschließend im Vollzug zu arbeiten“ (1999, S. 177). 
Der Ruf nach einer, aufgrund der Spezifika des Strafvollzugs und der inhaftierten Klientel (vgl. Kap. 4.2 und 4.3), gesonderten Qualifizierung der Lehrenden beginnt bereits im Ende des 19. Jahrhundert. So plädiert Krell schon 1874 dafür, dem neu Eintretenden eine erfahrene Person zuzuteilen. „Dadurch wird der Uebelstand vermieden, der für Lehrer und Schüler gleich nachtheilig ist, nämlich, dass jeder Neueintretende erst mehrere Jahre experimentiren muss, ehe er die Befähigung erlangt, von der allein sein Erfolg abhängt, und die er gleich mit ins Amt mitbringen sollte, die er aber, so wie eben die Dinge liegen, nicht mitbringen kann“"(1874, S. 58f.). Über 120 Jahre später hatten sich die Auffassungen unter dem Aspekt eines zielführenden Freiheitsentzugs, insbesondere bezüglich der pädagogischen Gesamtkonzeption zwar gewandelt, am Qualifizierungsdefizit der Lehrenden hatte sich jedoch nichts geändert. Folgerichtig merkte Prim deshalb kritisch an, dass die herkömmliche Lehrerbildung ,weder kriminologische noch vollzugspädagogische Kenntnisse [vermittelt, M. S.]. Schon gar nicht bereitet sie auf ein berufliches Handeln in einer streng hierarchischen, höchst komplexen Institution vor" (1989, S. 184).

Alles in allem muss demnach konstatiert werden, dass es "die spezifischen Klientele, deren Herkunft und soziale Situation sowie [...] die sehr schwierigen Kontextbedingungen" (Walkenhorst 2002a, S. 411) sind, welche die dominierenden Belastungen im Rahmen der Lehrerarbeit hinter Gittern darstellen.

\subsection{Die totale Institution als Dominante}

Gefängnisse besitzen, ebenso wie Kasernen, Klöster oder Internate, einen allumfassenden Charakter. Goffmann (1973) subsumiert diese daher unter dem Begriff der „totalen Institution“. Dabei geht er davon aus, dass totale Institutionen dadurch gekennzeichnet sind, dass ,die grundlegende Ordnung, nach der der einzelne an verschiedenen Orten schläft, spielt, arbeitet - und dies mit wechselnden Partnern, unter verschiedenen Autoritäten und ohne einen umfassenden rationalen Plan“" (ebd., S. 17), aufgehoben ist. Hinzu kommt, dass zwischen denen, welchen die Freiheit und Autorität entzogen wird und denen, die diesen Zustand verwalten und erhalten ,antagonistische Stereotypen" (ebd., S. 20) entstehen, welche wiederum durch die Logik der totalen Institution gefördert werden. In der Folge bilden sich ,zwei verschiedene soziale und kulturelle Welten [Subkulturen, M. S.], die mit einigen offiziellen Berührungspunkten nebeneinanderher bestehen, sich aber kaum gegenseitig durchdringen" (ebd.). Aus Sicht der Lehrenden stellt dies ein Problem dar, denn „die Subkultur schafft eine Frontenbildung zwischen den Inhaftierten und den Betreuern. Sie hat eine eigene Sprache, ihre eigenen Regeln, und ihr 
fällt es nicht schwer, alle Erziehungsansätze und Bemühungen im Keim zu ersticken“ (Wattenberg zit. nach Silber 2000, S. 57).

Paradoxer Weise sind nicht die Gesetze, welche den Strafvollzug regeln soll(t)en, die strukturgebende Instanz, sondern die Institution selbst. Für die Lehrenden im Vollzug ist dies nicht anders. Sie „,sind nur einen Teil ihres Lebens in der ,totalen Institution " eingebunden [...] Es geht dabei aber allein um die Arbeitskraft, nicht um den Menschen; es geht um den Dienst und nicht die Person (= Individualität). Auch die Mitarbeiter sind insofern Opfer der Konventionen der ,totalen Institution "“ (Hervorhebung im Original; Bammann 2010, S. 42). Das heißt die Totalität, wenn sie denn als allumfassende, einschränkende Eigenschaft verstanden wird, hat zwangsläufig Auswirkungen auf die Lehrerarbeit und die Schule als Gesamtheit. Konkret bedeutet dies, dass es in Bezug auf die Grundidee schulisch organisierter Bildung zu einem Paradigmenwechsel kommt (ausführlicher in Schweder 2014). Während Bildungsinstitutionen im öffentlichen Schulsystem eher dazu dienen geeignete Rahmenbedingungen für Bildung bereitzustellen, ordnet sich im Strafvollzug die Bildung den Rahmenbedingungen und damit dem Konzept der ,sicheren Verwahrung“ unter. Es geht demnach eben nicht darum, geeignete Rahmenbedingungen für das Lehren und Lernen zu schaffen, sondern für das Verwahren. In der Konsequenz kann weder die öffentliche Schul- und Unterrichtslogik noch die Lehrerarbeit selbst unreflektiert in den Strafvollzug kopiert werden (vgl. auch Silber 2000, S. 19). Insofern müssen nicht nur die Schulen im Vollzug, sondern ebenso die Lehrenden ,ein Selbstbewusstsein dahingehend entwickeln, dass ihre Prozesse zunächst einmal terminlich Vorrang haben und nicht beliebig Verfügungsmasse anderer, zumal schulfremder Stellen sind“" (Walkenhorst 2002b, S. 336).

Es bleibt festzustellen, dass es wichtig erscheint, sich über die Spezifika der totalen Institution und deren Auswirkungen (positive wie negative) auf die Lehrerarbeit bewusst zu sein, ohne den Strafvollzug als „Gegenwelt“ (Silber 1999, S. 57) zu verstehen. Inhaltlich ist dies auf die Inhaftierten übertragbar, welche sich als Adressaten zwar vom Lernenden außerhalb des Vollzugs unterscheiden, indes aber keine ,anderen Menschen“ sind.

\subsection{Die Straftäter als Subdominante}

Aus Sicht der Lehrenden spielen die anthropogenen Voraussetzungen der Adressaten im Rahmen der Planung und Gestaltung von Unterricht eine wesentliche Rolle. Im Allgemeinen kann festgestellt werden, dass die Gemeinsamkeit auf Seiten der Gefangen wohl einzig in der Heterogenität ${ }^{7}$ ihrer Le-

7 Matt und Maul (2005, S. 198f.) beschreiben die Heterogenität sehr anschaulich anhand der Kategorien: Vorbildung, Alter, Nationalität, Inhaftierungszeit, Drogenkonsum, Konzentrationsfähigkeit und Lernbereitschaft. 
bens- und Bildungsverläufe liegt. „Im Vergleich zu den Verhältnissen in Freiheit sind unter den Gefangenen Sonderschüler, vorzeitige Schulabgänger und Berufsausbildungsabgänger, Hilfs- und Gelegenheitsarbeiter überrepräsentiert" Hoffmann 1989, S. 466, siehe auch Reinheckel 2013). Hinzu kommen Defizite in der Persönlichkeit, das heißt der Fähigkeit mit Problemen, Misserfolgen, Aggressionen oder Ähnlichem umzugehen sowie ein unreflektierter und ausgeprägter Umgang mit Drogen. „Dies verdeutlicht nicht nur die Notwendigkeit für schulische Einrichtungen im Strafvollzug, sondern verweist auch auf die Schwierigkeiten der pädagogischen Aufgabe“ (ebd., ebenso Bierschwale et al. 2001, S. 171).

Oftmals haben die Lehrenden zu den komplexen Problemlagen der Gefangenen keinen Zugang, u. a. weil sie ihren Blick nur auf die defizitäre Schulbildung beschränken (vgl. Borchert 2007, S. 203) und es ihnen an „biografischer Erfahrungsnähe zu ihren Schülern“ (Prim 1989, S. 186) mangelt. Zudem können gewisse Informationen, z. B. zur Tat, so sie denn durch den Gefangenen in den Unterricht transportiert oder an den Lehrenden herangetragen werden, zur Belastung für den Lehrenden werden (vgl. Borchert 2007, S. 200ff.). Inwieweit die Kenntnis darüber einen individualisierten Unterricht oder aber Vorbehalte und Vorurteile befördern würde, ist schwer zu sagen. Allerdings stellt eben gerade das Merkmal Devianz, neben dem der Inhaftierung, eine typische Disposition der Klientel dar (Prim 1986, S. 300).

Ein weiterer, im Kontext der Lehrerarbeit nicht zu unterschätzender, Aspekt ist die Verlagerung der aus den institutionellen und subkulturellen Einflüssen heraus entstandenen Strukturen und Einstellungen ${ }^{8}$ in den Unterricht. Wie nachhaltig diese Anpassungsprozesse gegenüber der Institution sind, ist folgendem Selbstzeugnis zu entnehmen (Wolf zit. nach Harbordt 1967, S. 86): „Ich weiß jetzt bis ins kleinste, wie ich mich verhalten muß im Gefängnis. Ich weiß, was erlaubt ist und was verpönt, was erwünscht und was verboten ist; ich weiß, wie man Gesuche gut durchkriegt, wie man zu Vergünstigungen kommt, wie man sich schnell einen guten Posten erringt und beliebt macht, ich weiß, wie man fünf Jahre ,rumbringt" - ohne Arrest, ohne Meldung; wie man es anstellt, daß man begnadigt wird. Nur - das ist etwas, was ich nicht verwerten kann. [...] Denn ich bin ja erzogen worden fürs Gefängnis [...] Sperren Sie mich ein - und Sie werden bestimmt Ihre Freude an mir haben". Zu dieser Anpassung an die Konventionen der Institution kommt die unvermeidliche und ,überlebensnotwendige“ Einordnung in die Insassenkultur sowie die in diesem Zusammenhang immer wieder zu verteidigende Stellung innerhalb dieser. „Du hast nur den Großen markiert,

8 Mit der Inhaftierung unabdingbar verbunden, wenn auch in unterschiedlichem Maße, ist ein nicht intendierter Sozialisationsprozess, welcher sich in der Anpassung an die Regeln der Institution und die der Subkultur mit ihr eigenen Regeln und Normen, zeigt (vgl. Harbordt 1967, S. 84). Diese unweigerlich ablaufenden Prozesse werden unter dem Begriff der Prisonisierung (Clemmer 1958, Ortmann 1993) subsumiert (vgl. auch Kap. 4.2). 
so nach dem Motto, aber in Wirklichkeit hattest du selber Angst, weil du wusstest ja nicht, was er auf einmal macht" (Bereswill 2001, S. 269).

Die beschriebene Problematik, insbesondere ihre Unvermeidbarkeit, ist dem Gesetzgeber ebenso bewusst, indem er dazu ermahnt den „schädlichen Folgen des Freiheitsentzuges" ( $\$ 3$ StVollzG) entgegenzuwirken. Gerade deshalb können und dürfen die Lehrenden ihre Arbeit nicht in einem verkürzten Verständnis, nämlich in dem des Wissensvermittlers, sehen.

\section{Fazit}

Unter Berücksichtigung der historischen Genese des Freiheitsentzugs wird klar, warum die Lehrenden scheinbar immer noch „Fremdkörper“" in den Strafvollzugsanstalten sind. Denn obwohl das Konzept der Resozialisierung das der Sühne und Vergeltung ersetzt hat, muss konstatiert werden, dass der Strafvollzug prinzipiell auch ohne Unterricht und Lehrer funktioniert. Die Tatsache, dass dies ein Bruch mit den geltenden Gesetzen, welche Bildungsmaßnahmen vorschreiben, bedeuten würde sei dabei einmal außen vor gelassen. Hiermit soll nur deutlich gemacht werden, dass die Grundidee des Strafvollzugs eine andere ist. Dieser Logik muss sich die Lehrerarbeit mit allen Konsequenzen unterordnen. Denn dass ,dieses Arbeiten im Rahmen eines Freiheitsentzuges mit Strafcharakter stattfindet, daß also Übelzufügung Realität ist, was auch immer Strafrechtsexperten (sic!) argumentieren mögen, ist [...] eine Binsenweisheit“" (Busch 1991, S. 263). Hinzu kommt, dass die Lernenden, wie oben beschrieben, in aller Regel komplexe und vor allem individuelle Problemlagen aufweisen sowie in ihrer Eigenschaft als Gefangene Prozessen der Prisonisierung ausgeliefert sind, die es „draußen“ so nicht gibt.

Schlussendlich bleibt zu konstatieren, dass es aufgrund der Besonderheiten der totalen Institution Strafvollzug und der historisch nachweisbaren Sonderstellung der Lehrenden innerhalb dieser bis heute kein klares und vor allem objektives Bild über die Lehrerarbeit gibt. Dies zeigt sich u. a. an der immer noch andauernden Diskussion zum, bzw. dem Ruf nach, einem konstituierenden Berufsbild. Diesem Desiderat könnte mit Hilfe einer systematischen Erfassung der Bedingungen sowie der Anforderungen im Kontext der Lehrerarbeit hinter Gittern entgegengewirkt werden. Hierauf aufbauend wäre es möglich, das erforderliche Know-how und damit Ausbildungskonzeptionen für die Lehrenden abzuleiten.

Um mit dem Bild von Ellger (1922, S. 7) abzuschließen, demzufolge die (Lehr)Inhalte ,in gesundem nahrhaften Brot bestehen“ sollten, und dieses gleichzeitig etwas zu erweitern: „Gesundes und nahrhaftes Brot" kann nur gebacken werden, wenn die Backstube die technisch-organisatorischen Voraussetzungen zur Verfügung stellt, welche hierfür von Nöten sind. Wenn 
dieses Ideal nicht in Gänze gewährleistet werden kann, weil die Umgebung dies in ihrer Eigenschaft nicht zulässt oder gewisse Arbeitsmittel und Arbeitsmethoden nicht anwendbar sind, ist es eine Frage der Ausbildung des Bäckers, ob dieser unter den beschränkten Bedingungen dennoch in der Lage ist ein solches Brot zu backen. An eine personenbezogene Zutatenauswahl ist dabei noch gar nicht gedacht.

\section{Literatur}

Bammann, K. (2010): Kreativität und künstlerisches Gestalten als Durchbrechung der „Totalen Institution“. Dissertation Universität Bremen Online: http://elib.suub. uni-bremen.de/edocs/00101889-1.pdf. (22-03-2013)

Behringer, G. (1901): Die Gefängnisschule. Ein Überblick über die geschichtliche Entwicklung, den heutigen Stand und die Bedeutung des Schul- und Bildungswesens in Strafanstalten. Freiburg i. Br.: C. L. Hirschfeld.

Bereswill, M. (2001): „Schmerzen des Freiheitsentzuges“. Gefängniserfahrungen und Überlebensstrategien männlicher Jugendlicher und Heranwachsender in Strafhaft. In: Bereswill, M./Greve, W. (Hrsg.): Forschungsthema Strafvollzug. 1. Aufl. Baden-Baden: Nomos, S. 253-285.

Bierschwale, P. (1994): Die Pädagogische Abteilung. Zum Berufsbild der Lehrer im Justizvollzug des Landes Niedersachsen. In: ZfStrVo, 43. Bd., H. 4, S. 195-203.

Bierschwale, P./Detmer, B./Pendon, M./Weidenhiller, M. (2001): Lehrerinnen und Lehrer im Justizvollzug. Beschreibung eines pädagogischen Arbeitsfeldes. In: Bundesarbeitsgemeinschaft der Lehrer im Justizvollzug (Hrsg.): Justizvollzug \& Pädagogik, Tradition und Herausforderung. 2. Auflage, Herbolzheim: Centaurus.

Blochmann, E./Geißler, G./Nohl, H./Weniger, E. (Hrsg.) (1962): Pestalozzis Gedanken zur Kriminalpädagogik. Weinheim: Beltz.

Bondy, C. (1997): Pädagogische Probleme im Jugend-Strafvollzug. Lüneburg: Ed. Erlebnispädagogik.

Borchert, J. (2007): Schule und Sozialarbeit im sächsischen Strafvollzug. Leipzig: Leipziger Univ.-Verlag.

Bundesarbeitsgemeinschaft der Lehrer im Justizvollzug (Hrsg.) (2001): Justizvollzug \& Pädagogik, Tradition und Herausforderung. 2. Auflage, Herbolzheim: Centaurus.

Busch, M. (1991): Der Lehrer im Strafvollzug - Ansprüche und Wirklichkeit im Berufsalltag. In: ZfStrVo, 50. Bd., H. 5, S. 259-264.

Clemmer, D. (1958): The prison community. New York: Holt, Rinehart and Winston.

Cornel, H. (1984): Geschichte des Jugendstrafvollzugs. Ein Plädoyer für seine Abschaffung. Weinheim und Basel: Beltz.

Deimling, G. (1969): Theorie und Praxis des Jugendstrafvollzugs in pädagogischer Sicht. Neuwied am Rhein und Berlin: Luchterhand.

Deimling, G. (1971): Zum Berufsbild des Lehrers an Justizvollzugsanstalten. In: Recht der Jugend und des Bildungswesens 19, S. 53-60. 
Eberle, H.-J. (1980): Lernen im Justizvollzug. Voraussetzungen und Ansätze einer Justizvollzugspädagogik und ihrer Didaktik. Frankfurt a. M.: Haag + Herchen.

Eberle, H.-J. (1983): Schule hinter Mauern: Die deutsche Gefängnisschule in ihrer geschichtlichen Entwicklung. In: Zeitschrift für Berufs- und Wirtschaftspädagogik, 79. Bd., H. 6, S. 439-449.

Eberle, H.-J. (1984): Lehrer im Strafvollzug: Zur Geschichte und gegenwärtigen Problematik. In: Sonderpädagogik, 14. Bd., H. 2, S. 63-73.

Ellger, H. (1922): Der Erziehungszweck im Strafvollzug. Halle: Marhold.

Foucault, M. (1994): Überwachen und Strafen. Die Geburt des Gefängnisses. Frankfurt a. M.: Suhrkamp. (erste dt. Auflage 1976)

Gesetz über den Vollzug der Freiheitsstrafe und der freiheitsentziehenden Maßregeln der Besserung und Sicherung (Strafvollzugsgesetz - StVollzG) Online: http:// www.gesetze-im-internet.de/bundesrecht/stvollzg/gesamt.pdf (22-03-2013).

Goffman, E. (1973): Asyle. Über die soziale Situation psychiatrischer Patienten und anderer Insassen, Frankfurt a. M.: Suhrkamp.

Harbordt, S. (1967): Die Subkultur des Gefängnisses. Eine soziologische Studie zur Resozialisierung. Stuttgart: Ferdinand Enke Verlag.

Hermann, W. (1926): Das Hamburgische Jugendgefängnis Hahnöfersand. Ein Bericht über die Erziehungsarbeit im Strafvollzug. Mannheim, Berlin, Leipzig: J. Bensheimer.

Hoffmann, M. (1989): Unterricht im Strafvollzug. In: Neukäter, H./Goetze, H. (Hrsg.): Handbuch der Sonderpädagogik. Pädagogik bei Verhaltensstörungen. Bd. 6, Berlin: Marhold, S. 464-472.

Krell, A. (1874): Aphorismen über das Unterrichtswesen in Straf-Anstalten. Ein Buch für Beamte an Straf-Anstalten, Bezirks- und Gerichtsgefängnissen, Pädagogen und Lehrer, sowie für Alle, die sich für Unterricht und Erziehung interessieren. Görlitz: H. Wollman's Verlag.

Marzahn, C./Ritz, H. (Hrsg.) (1984): Zähmen und Bewahren. Die Anfänge bürgerlicher Sozialpolitik. Bielefeld: AJZ.

Matt, E./Maul, K. (2005): Das Problem der Heterogenität der Klientel für Bildungsmaßnahmen einer Justizvollzugsanstalt. In: ZfStrVo, 54. Bd., H. 4, S. 198-202.

Müllges, U./Justin, J. J. (1996): Erziehung und Bildung. Analysen ihrer Theorie und Wirklichkeit. Frankfurt a. M., Berlin, Bern; New York, Paris, Wien: Lang.

Myschker, N./Hoffmann, M. (1984): Unterricht mit jugendlichen Inhaftierten. Hagen (Fernuniversität).

Ortmann, R. (1993): Prisonisierung. In: Kaiser, G./Kerner, H.-J./Sack, F./Schellhoss, H. (Hrsg.): Kleines Kriminologisches Wörterbuch. Heidelberg: C. F. Müller/ UTB, S. 402-409.

Pestalozzi, J. H. (1785): Lienhard und Gertrud. Ein Buch fürs Volk. Dritter Theil, § 76. In: Blochmann, E./Geißler, G./Nohl, H./Weniger, E. (Hrsg.) (1962): Pestalozzis Gedanken zur Kriminalpädagogik. Eingeleitet von Franz Zeugner. 2. Auflage, Weinheim: Verlag Julius Belz.

Prim, R. (1986): Der gefangene Schüler und die Lebenswelt des Gefängnisses als berufspädagogische Herausforderung. In: Zeitschrift für Berufs- und Wirtschaftspädagogik, 82. Bd., H. 4, S. 299-311.

Prim, R. (1989): Probleme und Chancen der Lehrer-Schüler-Beziehung im Behandlungsvollzug. In: Siegfried, B. (Hrsg.): Der Umgang mit Schülerkriminalität in der Praxis. Bd. 2, Stuttgart: Verl. für Angewandte Psychologie, S. 184-205. 
Reinheckel, S. (2008). Schulische Bildung im deutschen Jugendstrafvollzug vom 19. Jh. bis in die Gegenwart - ein kurzer Rückblick. In: Forum Strafvollzug, 5. Bd., S. 205-210.

Rheinheckel, S. (2013): Geringqualifikation bei männlichen Strafgefangenen im geschlossenen Jugendstrafvollzug der Bundesrepublik Deutschland - eine empirische Untersuchung. Forschungsbericht, Berlin. Online: http://www.ssoar.info/ ssoar/handle/document/34941 (03.03.2014).

Rudow, B. (2000): Arbeits- und Gesundheitsschutz im Lehrerberuf. Schulmanagement, 31. Bd., H. 6, S. 27-31.

Schweder, M. (2014): Lehrer sein, das ist schwer - Hinter Gittern noch viel mehr?! In: Die berufsbildende Schule (BbSch) Jg. 66, H. 3, S. 103-107.

Silber, I. (2000): Negative Jugendkarrieren und pädagogische Interventionen: Abweichende Lebensgeschichten und Lernprozesse im Strafvollzug. Heidelberg: Winter (Edition S).

Wagner, G. (1984): Das absurde System. Strafurteil und Strafvollzug in unserer Gesellschaft. Heidelberg: C. F. Müller Juristischer Verlag.

Walkenhorst, P. (2002a): Bestandsaufnahme und Perspektiven zur Schule im Jugendstrafvollzug. In: DVJJ-Journal, 13. Bd., H. 4, S. 404-416.

Walkenhorst, P. (2002b): „Gute Schulen“ im Jugendstrafvollzug - Jugendstrafvollzug als ,gute Schule“ - Überlegungen zu Voraussetzungen und Möglichkeiten. In: Bereswill, M./Höynck, T. (Hrsg.): Jugendstrafvollzug in Deutschland - Grundlagen, Konzepte, Handlungsfelder: Beiträge aus Forschung und Praxis. Mönchengladbach: Godesberg, S. 319-355.

Wichern, J. H. (1860): Erlaß wegen des Schulunterrichts in den Strafanstalten (1860). In: Meinhold, P. (1973): Johann Hinrich Wichern. Sämtliche Werke. Bd. 6, Hamburg: Lutherisches Verlagshaus, S. 179-188.

Wittig, K. (1939): Das Werden der deutschen Gefängnisschule. Eine Sammlung von Zeugnissen über sie. In: Blätter für Gefängniskunde: Organ der Deutschen Gesellschaft für Gefängniskunde in der Akademie für Deutsches Recht, (70), S. 84177. 